\title{
Ethnic Differences in Stroke Recurrence
}

\author{
Lynda D. Lisabeth, PhD, ${ }^{1,2}$ Melinda A. Smith, MPH, ${ }^{2}$ Devin L. Brown, MD, MS, ${ }^{2}$ \\ Lemuel A. Moyé, MD, PhD, ${ }^{3}$ Jan M. H. Risser, $\mathrm{PhD},{ }^{3}$ and Lewis B. Morgenstern, $\mathrm{MD}^{1,2}$
}

Objective: To determine whether stroke recurrence and the effect of recurrence on mortality differ by ethnicity.

Methods: Using methods from the Brain Attack Surveillance in Corpus Christi project, we prospectively identified first-ever ischemic strokes from emergency department logs and hospital admissions (January 2000 to December 2004). Recurrent strokes and deaths were identified for the same period. Cumulative probability of stroke recurrence was estimated. Cox proportional hazards models were used to examine ethnic differences in recurrence and to examine the relation among ethnicity, recurrence, and mortality.

Results: During the time interval, 1,345 first-ever ischemic strokes were validated. Median age of patients was 72 years; 53\% were Mexican American (MA). There were 126 recurrent strokes. Cumulative risk for recurrence at 30 days and 1 year was 2.6 and 7.5\%, respectively. MAs had higher risk for stroke recurrence (risk ratio, 1.57; 95\% confidence interval, 1.05-2.34) compared with non-Hispanic white patients, adjusted for demographics, stroke risk factors, and stroke severity. Stroke recurrence was related to mortality to a similar extent across ethnic groups (non-Hispanic white patients: risk ratio, 3.32; 95\% confidence interval, 2.07-5.32; MAs: risk ratio, 2.35; 95\% confidence interval, 1.42-3.88).

Interpretation: MAs had higher stroke recurrence risk compared with non-Hispanic white patients. Stroke recurrence had an important impact on mortality. Efforts to reduce stroke recurrence in MAs are needed.

Ann Neurol 2006;60:469-475

The burden of stroke encompasses both first-ever and recurrent events. Each year, 700,000 people in the United States experience strokes, and roughly 200,000 of these strokes occur in individuals with a previous stroke history. ${ }^{1}$ In the United States, the majority of strokes are ischemic in nature. Estimates of 30-day risk for recurrent stroke after an initial ischemic stroke are 2 to $4 \%,{ }^{2-5}$ and estimates of 1 -year risk for recurrence range from 7 to $13 \% .^{3,5-9}$ Recurrent strokes are associated with worse functional outcome $e^{4}$ compared with first-ever strokes and also with an increased risk for death. ${ }^{7,10-12}$

Hispanic individuals in the United States experience a greater burden of stroke compared with nonHispanic white individuals (NHWs) and may be at higher risk of stroke recurrence. ${ }^{13-15}$ Mexican Americans (MAs), the largest and fastest growing subgroup of Hispanic Americans, have been shown to have an increased risk for ischemic stroke compared with NHWs, especially at younger ages. ${ }^{14}$ Other aspects of stroke burden that have been shown to differ between MAs and NHWs include risk factor profiles ${ }^{16}$ and survival after stroke. ${ }^{17}$ Risk for stroke recurrence and the influence of recurrence on subsequent mortality have not been investigated in the MA population. The goals of this study were: (1) to estimate stroke recurrence after initial ischemic stroke in a biethnic population; (2) to determine whether stroke recurrence differs by ethnicity; (3) to estimate the effect of stroke recurrence on all-cause mortality after stroke; and (4) to determine whether the effect of stroke recurrence on mortality differs by ethnicity.

\section{Subjects and Methods}

This was a primary, prespecified analysis of the Brain Attack Surveillance in Corpus Christi (BASIC) project. BASIC is a population-based stroke surveillance project designed to capture all strokes in Nueces County, a geographically isolated, urban, biethnic community on the southeast coast of Texas. Nueces County has a population of 313,645 and is 56\% MA and $38 \%$ NHW. The county, which serves as a regional referral medical center for southeast Texas, is approximately 150 miles from Houston and San Antonio, the two closest cities. BASIC methodology has been described previously. ${ }^{14,18}$ In brief, between January 1, 2000, and December 31, 2004, trained abstractors, using validated screening procedures, identified ischemic stroke cases through rigorous active and passive surveillance of emergency department logs, hospital admissions records, and discharge diagnosis codes, regardless of the date of presentation. In addition, an out-of-hospital sampling frame including 47 of 167 primary care physicians,
From the ${ }^{1}$ Department of Epidemiology, University of Michigan School of Public Health; ${ }^{2}$ Stroke Program, University of Michigan Medical School, Ann Arbor, MI; and ${ }^{3}$ University of Texas Health Science Center at Houston School of Public Health, Houston, TX.

Received May 23, 2006, and in revised form Jun 19. Accepted for publication Jun 30, 2006.
Published online Aug 22, 2006, in Wiley InterScience (www.interscience.wiley.com). DOI: 10.1002/ana.20943

Address correspondence to Dr Lisabeth, Department of Epidemiology, University of Michigan School of Public Health, 109 South Observatory, Ann Arbor, MI 48109-2029.

E-mail: llisabet@umich.edu 
4 of 11 nursing homes, and all 11 neurologists in Nueces County was used to identify nonhospitalized stroke cases. Using previously published criteria, ${ }^{19}$ BASIC project neurologists, blinded to subject ethnicity and age, validated cases as stroke or no stroke. Excluded were patients younger than 45 years, those with traumatic strokes, those who were not Nueces County residents (based on zip code), and for this analysis, those with a history of stroke/transient ischemic attack determined from the medical record. The final sample size for the analysis was 1,345 patients.

\section{Recurrent Stroke Events}

The time period for identifying recurrent events was the same as for index events. All hospital admissions and emergency department visits for strokes subsequent to the index event were captured using the case-ascertainment methods described earlier. Recurrent stroke was defined as a newly validated ischemic stroke, intracerebral hemorrhage, or subarachnoid hemorrhage occurring after the index event. Because recurrent strokes were identified from subsequent hospital admissions and emergency department visits, it is possible that a new stroke occurring within the initial hospitalization was not detected, resulting in an underestimation of early risk for recurrence; however, the magnitude of this bias is likely small. In individuals with multiple recurrent strokes, only the first recurrence was considered.

Because it is possible that recurrent strokes resulting in death may be more likely to be missed by routine surveillance, resulting in an underascertainment of the recurrence end point, we investigated the magnitude of such bias. Specifically, we examined the frequency of recurrent strokes identified from review of mortality/cause of death data not captured by surveillance methods using a convenience sample of deaths among ischemic stroke cases. The sample included the first 100 deaths among MA and NHW ischemic stroke cases beginning January 1, 2000. Medical records were reviewed by a study neurologist (L.M.) to determine the underlying cause of death. ${ }^{20,21}$ If the cause of death was found to be stroke, it was then determined whether the stroke resulting in death was previously captured through the BASIC active and passive surveillance methodology. Among the 100 mortality cases reviewed, 5 cases had insufficient data to determine underlying cause of death (2 MAs; 3 NHWs). Among cases with sufficient data, there was 1 (2.6\%) recurrent stroke identified among MA cases $(n=39)$ that was not captured by routine surveillance and 2 recurrent strokes identified (3.6\%) among NHW cases $(\mathrm{n}=56)$, with no significant ethnic difference, suggesting that estimates of recurrence risk should be minimally impacted by this issue.

\section{Ethnicity, Stroke Risk Factors, and Stroke Severity}

Ethnicity and risk factors were obtained from the medical records. We have previously demonstrated high agreement between ethnicity recorded in the medical record and selfreported ethnicity in this population $(\kappa=0.94) .{ }^{22}$ Risk factors included history of hypertension, high cholesterol, diabetes mellitus, atrial fibrillation, smoking (ever vs never), and coronary artery disease. If the medical records specifically reported either a positive or no history of any of these risk factors, they were so recorded in the abstraction. If the med- ical records contained no mention of a specific risk factor, that risk factor was recorded as absent in the abstraction. We have previously demonstrated high agreement between selfreport and medical record risk factor data for most factors (atrial fibrillation: percentage agreement $=82.1 \%, \kappa=$ 0.54; diabetes: percentage agreement $=91.6 \%, \kappa=0.86$; coronary heart disease: percentage agreement $=82.3 \%, \kappa=$ 0.63 ; hypertension: percentage agreement $=82.9 \%, \kappa=$ 0.62 ; high cholesterol: percentage agreement $=67.1 \%, \kappa=$ 0.37). ${ }^{16}$ To determine initial stroke severity, we retrospectively calculated the National Institutes of Health Stroke Scale (NIHSS) score from data abstracted from the medical record in accordance with the validated method of Williams and colleagues. ${ }^{23}$

\section{Mortality}

Mortality from all causes was ascertained through active and passive surveillance of in-hospital strokes and through examination of Texas Department of Health death certificate data for the state of Texas for the period January 1, 2000, through December 31, 2004. One year was allowed to pass before accessing the vital statistics data to ensure nearcomplete capture of deaths. Personal identifiers (first and last names, social security number, date of birth, and permanent address) from the stroke cases were compared with those in the Texas Department of Health death certificate database. Linking of at least three of the five identifiers was required for the BASIC stroke case to be considered a match to the mortality data.

\section{Statistical Analysis}

Survival times (days) were calculated from the date of first presentation of the index ischemic stroke case to the time of the first recurrent stroke or death. For the stroke recurrence end point, cases were censored at time of death or the end of the follow-up period (December 31, 2004) for patients who remained alive but without recurrence as of the end of the follow up. For the mortality end point, patients who were still alive were censored at the end of the follow-up period (December 31, 2004). Death from all causes was considered. To examine differences in baseline risk factors between MAs and NHWs, we calculated odds ratios (ORs) and 95\% confidence intervals (CIs). Kaplan-Meier survival methods were implemented to estimate cumulative probability of stroke recurrence after initial ischemic stroke. Stroke recurrence in MAs was compared with that in NHWs with a log-rank test. We also compared stroke recurrence by ethnicity stratified by age group (45-59, 60-74, and $\geq 75$ years). Multivariable regression using a Cox proportional hazards model was used to examine ethnic differences for stroke recurrence adjusted for potential confounders. Variables were prespecified for inclusion into the multivariable model and included demographics (age [modeled continuously] and sex) and stroke risk factors (atrial fibrillation, hypertension, diabetes, coronary artery disease, high cholesterol, smoking, and NIHSS [modeled continuously and then as indicator variables based on quartiles in a separate model]). The final sample size for the multivariable model was 1,311 (124 recurrent strokes) due to 33 cases with missing information regarding smoking status 
and 1 case with missing information on coronary artery disease.

A Cox proportional hazards model was constructed with stroke recurrence modeled as a time-dependent covariate to examine the association of stroke recurrence with all-cause mortality. Variables included in the model were prespecified and included ethnicity, age (modeled continuously), sex, stroke severity (NIHSS modeled continuously and then as indicator variables based on quartiles), atrial fibrillation, hypertension, diabetes, coronary artery disease, smoking, and high cholesterol. The final sample size for the multivariable model was 1,308 (403 deaths) due to cases with missing information regarding smoking status $(\mathrm{n}=33)$, coronary artery disease $(\mathrm{n}=1)$, and NIHSS $(\mathrm{n}=3)$. To determine whether the association of recurrence with all-cause mortality differs by ethnicity, we reran the above model stratified by ethnicity.

All analysis was conducted in SAS (SAS Institute, Cary, NC). The project was approved by the University of Michigan Institutional Review Board and each of the Nueces County hospitals.

\section{Results}

Abstractors screened 21,960 cases between January 1, 2000, and December 31, 2004. Of these cases, 4,775 met screening criteria and were reviewed by a study neurologist for validation. There were 4,087 validated cases of cerebrovascular disease during the study time period. Two hundred twenty-five African American cases, 18 Asian/Pacific Islander cases, and 32 cases with unknown race/ethnicity were excluded from the analysis due to small sample size, leaving 3,812 validated cerebrovascular events (ischemic stroke, transient isch- emic attack, intracerebral hemorrhage, subarachnoid hemorrhage). All first-ever ischemic strokes $(\mathrm{n}=$ $1,345)$ were included in the final baseline population.

Median age of the 1,345 ischemic stroke cases was 72.0 years (inner quartile range, 61.0-80.0). Fiftythree percent of the cases were MA $(n=715)$, and $52.0 \%(\mathrm{n}=700)$ were female. MAs were significantly younger than NHWs (Table 1) and were more likely to have diabetes (OR, 3.50; 95\% CI, 2.76-4.44). NHWs were more likely to have atrial fibrillation (OR, $1.89 ; 95 \% \mathrm{CI}, 1.34-2.67)$ and more likely to smoke (OR, 1.48; 95\% CI, 1.18-1.87) compared with MAs.

\section{Stroke Recurrence}

Median length of follow-up for the stroke recurrence end point was 534 days (inner quartile range: 1391,079). There were 126 recurrent strokes during the follow-up period. Of those 126 strokes, 118 (93.7\%) were ischemic strokes (MA, 76; NHW, 42) and 8 (MA, 5; NHW, 3) were intracerebral hemorrhages. There was no significant difference in the distribution of recurrent stroke type by ethnicity. There were no cases of subarachnoid hemorrhage among the recurrent strokes.

Cumulative risk for recurrent stroke at 30 days, 90 days, 1 year, and 2 years was 2.6, 3.9, 7.5, and 11.2\% respectively. Survival free from recurrent stroke is displayed in Figure 1 by ethnicity, with numerical data summarizing cumulative risk for stroke recurrence at select time points presented in Table 2. Recurrent stroke risk differed significantly by ethnicity $(p=$

Table 1. Demographics and Risk Factor Profiles by Ethnicity $(n=1,345)$ : The Brain Attack Surveillance in Corpus Christi (BASIC) Project, Nueces County, Texas, January 2000 to December 2004

\begin{tabular}{|c|c|c|c|c|c|}
\hline \multirow[b]{2}{*}{ Characteristics } & \multicolumn{2}{|c|}{$\mathrm{MA}(\mathrm{N}=715)$} & \multicolumn{2}{|c|}{ NHW $(\mathrm{N}=630)$} & \multirow[b]{2}{*}{ OR (95\% CI) } \\
\hline & $\mathrm{n}$ & $\%$ & $\mathrm{n}$ & $\%$ & \\
\hline \multicolumn{6}{|l|}{ Age, yr } \\
\hline $45-59$ & 191 & 26.7 & 96 & 15.2 & 1.00 \\
\hline $60-74$ & 286 & 40.0 & 202 & 32.1 & $0.71(0.52-0.96)$ \\
\hline$\geq 75$ & 238 & 33.3 & 332 & 52.7 & $0.36(0.27-0.48)$ \\
\hline Female sex & 371 & 51.9 & 329 & 52.2 & $0.99(0.80-1.22)$ \\
\hline Hypertension & 505 & 70.6 & 428 & 67.9 & $1.14(0.90-1.43)$ \\
\hline High cholesterol & 159 & 22.2 & 132 & 21.0 & $1.08(0.83-1.40)$ \\
\hline Atrial fibrillation & 60 & 8.4 & 93 & 14.8 & $0.53(0.38-0.75)$ \\
\hline Coronary artery disease & 205 & 28.7 & 189 & 30.0 & $0.94(0.74-1.19)$ \\
\hline Diabetes & 364 & 50.9 & 144 & 22.9 & $3.50(2.76-4.44)$ \\
\hline Smoking ${ }^{\mathrm{a}}$ & 199 & 28.3 & 225 & 37.0 & $0.67(0.53-0.85)$ \\
\hline \multicolumn{6}{|l|}{ NIHSS score ${ }^{b}$} \\
\hline $0-1$ & 171 & 24.0 & 152 & 24.2 & 1.00 \\
\hline $2-3$ & 183 & 25.7 & 168 & 26.8 & $0.97(0.72-1.31)$ \\
\hline $4-7$ & 172 & 24.1 & 128 & 20.4 & $1.19(0.87-1.64)$ \\
\hline$>7$ & 187 & 26.2 & 180 & 28.7 & $0.92(0.68-1.25)$ \\
\hline
\end{tabular}

${ }^{a}$ Thirty-three cases with missing data.

${ }^{\mathrm{b}}$ Four cases with missing data.

MA = Mexican American; NHW = non-Hispanic white; OR = odds ratio comparing MAs with NHWs; CI = confidence interval; NIHSS $=$ National Institutes of Health Stroke Scale. 


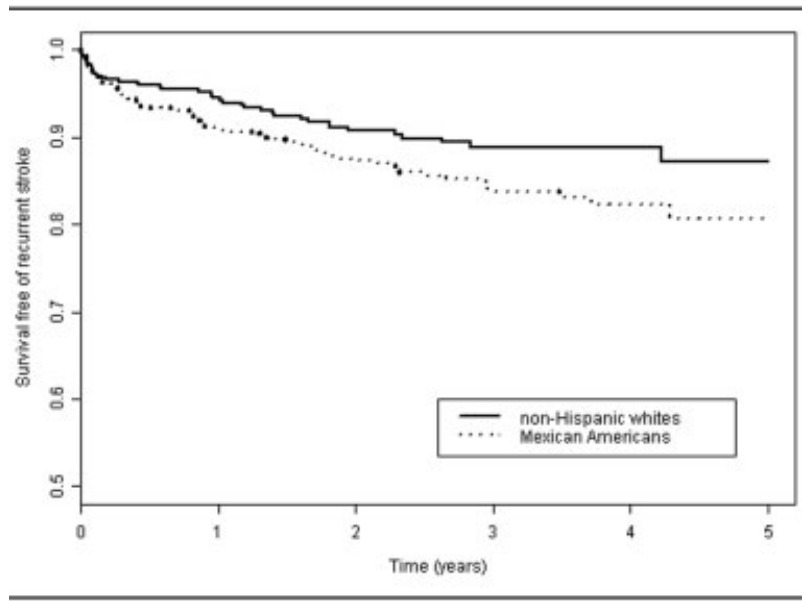

Fig 1. Survival free of recurrent stroke by ethnicity $(n=$ 1,345). The Brain Attack Surveillance in Corpus Christi (BASIC) project, Nueces County, Texas, January 2000 to December 2004. Dashed line represents Mexican American individuals; solid line represents non-Hispanic white individuals.

0.0311, log-rank test), with MAs having higher recurrence risk (see Fig 1). Cumulative risk for recurrent stroke by ethnicity, stratified by age group, is displayed in Figure 2 and summarized in Table 2. From Figure 2 , it appears that ethnic differences in recurrence may be largest at younger ages. There were no significant ethnic differences in recurrence within age groups, although the number of events within each stratum was small (age 45-59 years: 31 events [MA, 25; NHW, 6]; age 60-74: 45 events [MA, 29; NHW, 16]; age $\geq 75$ : 50 events (MA, 27; NHW, 23).

In the unadjusted Cox model for recurrence, the relative risk for recurrence comparing MAs with NHWs was 1.49 (95\% CI, 1.03-2.14). In the multivariable model, with adjustment for age, sex, atrial fibrillation, diabetes, hypertension, coronary artery disease, high cholesterol, smoking, and NIHSS, the risk ratio (RR) for ethnicity increased slightly and remained significant (RR, 1.57; 95\% CI, 1.05-2.34). Modeling NIHSS as indicator variables based on quartiles of the distribution did not change the results for ethnicity (RR, 1.55; 95\% CI, 1.04-2.32). Results from the multivariable model are included in Table 3.

\section{Stroke Recurrence and Mortality}

Median length of follow-up for the mortality end point was 597 days (inner quartile range, 170-1,136). There were $417(31.0 \%)$ deaths from all causes during the follow-up period. Stroke recurrence was significantly associated with risk for death in bivariate analysis (RR, 2.55; 95\% CI, 1.83-3.55). Stroke recurrence remained significant in the multivariable model (RR, 2.67; 95\% CI, 1.90-3.76). When the model was rerun using indicator variables for NIHSS based on quartiles of the distribution, the parameter estimate for stroke recurrence attenuated slightly (RR, 2.45; 95\% CI, $1.74-$ $3.45)$. Results from the full model are included in Table 4 . In the stratified analysis, the effect of recurrence on mortality did not differ significantly by ethnicity (NHWs: RR, 3.32; 95\% CI, 2.07-5.32; MAs: RR, 2.35; $95 \%$ CI, 1.42-3.88).

\section{Discussion}

This study found a significantly higher risk for recurrent stroke among MAs compared with NHWs. Results from a study conducted in a large urban hospital also found that Hispanic individuals of Puerto Rican heritage had a nearly threefold increased risk for recurrence compared with white individuals, although sample sizes within ethnic groups were small. ${ }^{15}$ Factors that could explain the observed ethnic difference include ethnic differences in underlying disease pathology, risk factor profiles, medication use and/or compliance, and genetic factors. If future work confirms the current finding that Hispanic individuals experience greater stroke recurrence risk compared with NHWs, factors contributing to ethnic differences should be investigated to improve outcomes after incident stroke. Our estimates of risk for recurrent stroke agree with previous estimates from prospective studies, lending confidence to our recurrence risk estimates. ${ }^{2,3,6-9,24,25}$ Furthermore, to avoid bias, we did not exclude early recurrences and we did include all types of recurrent stroke, ischemic or hemorrhagic; $94 \%$ of recurrent events were ischemic.

Recurrent stroke was strongly associated with allcause mortality and influenced all-cause mortality risk

Table 2. Cumulative Risk (\%) for Stroke Recurrence by Ethnicity and Age for Select Time Points ( $n=1,345)$ : The Brain Attack Surveillance in Corpus Christi (BASIC) Project, Nueces County, Texas, January 2000 to December 2004

\begin{tabular}{|c|c|c|c|c|c|c|c|c|c|c|c|c|}
\hline \multirow{3}{*}{$\begin{array}{l}\text { Age } \\
\text { Group, } \\
\text { yr }\end{array}$} & \multicolumn{12}{|c|}{ Time Period } \\
\hline & \multicolumn{4}{|c|}{30 Days } & \multicolumn{4}{|c|}{90 Days } & \multicolumn{4}{|c|}{1 Year } \\
\hline & $45-59$ & $60-74$ & $\geq 75$ & All Ages & $45-59$ & $60-74$ & $\geq 75$ & All Ages & $45-59$ & $60-74$ & $\geq 75$ & All Ages \\
\hline MA & 4.3 & 1.8 & 2.8 & 2.8 & 6.6 & 2.6 & 4.3 & 4.2 & 9.7 & 8.4 & 9.7 & 9.1 \\
\hline NHW & 2.2 & 2.6 & 2.0 & 2.3 & 3.3 & 4.3 & 2.8 & 3.4 & 4.6 & 5.0 & 6.5 & 5.5 \\
\hline
\end{tabular}

$\mathrm{MA}=$ Mexican American; NHW $=$ non-Hispanic white. 

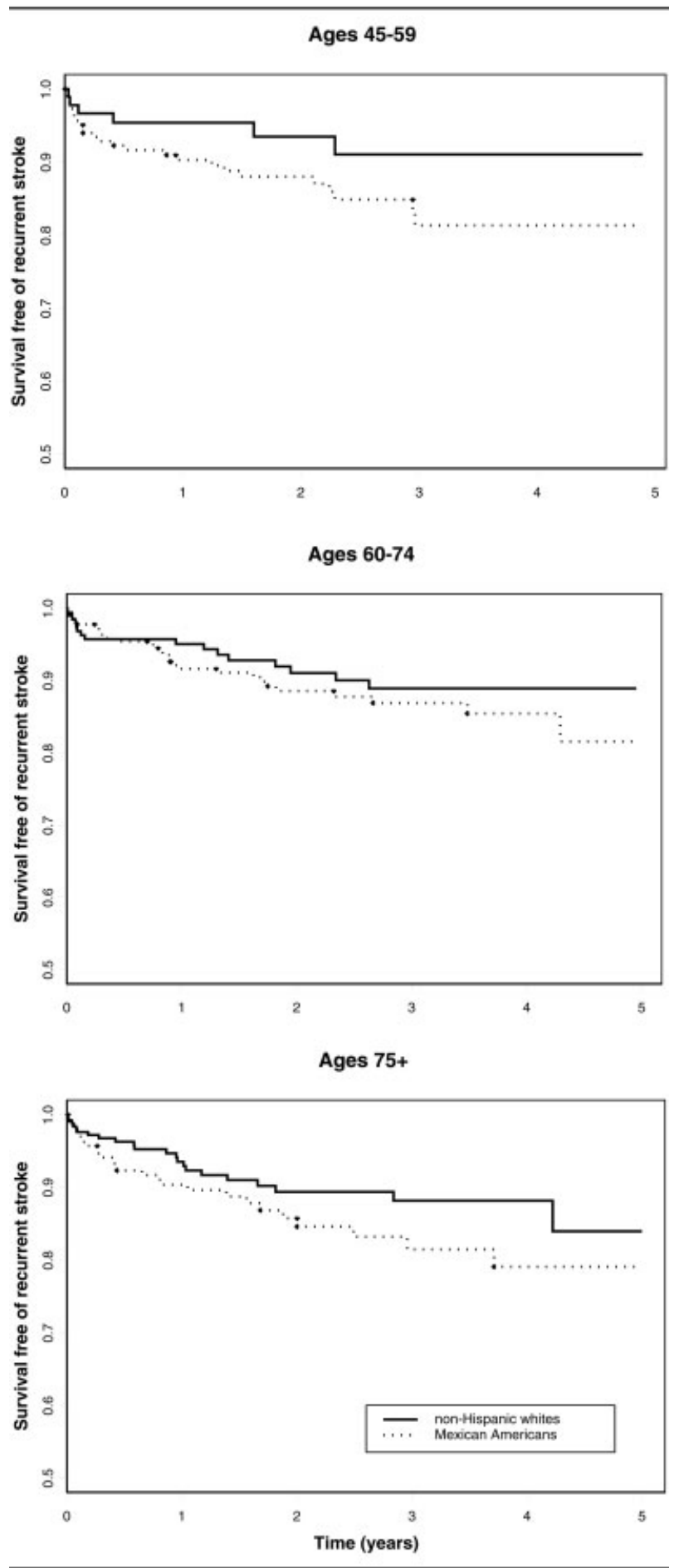

Fig 2. Survival free of recurrent stroke by ethnicity and age group $(n=1,345)$. The Brain Attack Surveillance in Corpus Christi (BASIC) project, Nueces County, Texas, January 2000 to December 2004. Dashed line represents Mexican American individuals; solid line represents non-Hispanic white individuals.

to a similar extent in both ethnic groups. Few studies have quantified the magnitude of increased mortality risk associated with stroke recurrence. One study reported a ninefold increased risk for a combined end
Table 3. Multivariable Model of Ethnicity in Relation to Stroke Recurrence $(n=1,311)$ : The Brain Attack Surveillance in Corpus Christi (BASIC) Project, Nueces County, Texas, January 2000 to December 2004

\begin{tabular}{lcc}
\hline Characteristics & RR & $95 \%$ CI \\
\hline Ethnicity (MA vs NHW) & 1.57 & $1.05-2.34$ \\
Age & 1.01 & $0.99-1.03$ \\
Sex (female vs male) & 1.13 & $0.78-1.64$ \\
Atrial fibrillation & 1.26 & $0.70-2.27$ \\
Hypertension & 1.12 & $0.74-1.70$ \\
Diabetes & 1.35 & $0.91-1.98$ \\
Coronary artery disease & 1.10 & $0.73-1.66$ \\
Smoking & 1.08 & $0.72-1.62$ \\
High cholesterol & 1.10 & $0.72-1.67$ \\
NIHSS & 0.99 & $0.96-1.03$
\end{tabular}

$\mathrm{RR}=$ relative risk; $\mathrm{CI}=$ confidence interval; $\mathrm{MA}=$ Mexican American; NHW = non-Hispanic white; NIHSS = National Institutes of Health Stroke Scale.

point of death or disability at 5 years among first-ever stroke cases due to stroke recurrence. ${ }^{12}$ Another study reported risk for death at 12 months to be 57 and 29\% in those with and without recurrence, respectively, in a cohort of first-ever ischemic stroke patients. ${ }^{7}$ Similarly, another study found that mortality was nearly double in patients with a recurrent stroke compared with those with a first-ever stroke. ${ }^{11}$ The analysis of stroke recurrence in relation to mortality is not straightforward because an early death prevents an individual from having a recurrent stroke. To avoid overestimating the effect of recurrence on mortality risk, we used a timedependent analysis of the recurrent event. We found recurrent stroke to confer a twofold to threefold increased risk for all-cause mortality adjusted for confounders and other independent predictors. The magnitude of this association was considerably larger than other predictors of death in this population including

Table 4. Multivariable Model of Stroke Recurrence in Relation to All-Cause Mortality $(n=1,308)$ : The Brain Attack Surveillance in Corpus Christi (BASIC) Project, Nueces County, Texas, January 2000 to December 2004

\begin{tabular}{lcc}
\hline Characteristics & RR & $95 \%$ CI \\
\hline Recurrent stroke & 2.67 & $1.90-3.76$ \\
Age & 1.06 & $1.04-1.07$ \\
Sex (female vs male) & 1.08 & $0.87-1.34$ \\
Ethnicity (MA vs NHW) & 0.66 & $0.53-0.82$ \\
NIHSS & 1.09 & $1.08-1.10$ \\
Atrial fibrillation & 1.05 & $0.81-1.38$ \\
Hypertension & 0.87 & $0.70-1.07$ \\
Diabetes & 1.33 & $1.06-1.66$ \\
Coronary artery disease & 1.30 & $1.05-1.60$ \\
Smoking & 1.13 & $0.89-1.44$ \\
High cholesterol & 0.60 & $0.45-0.80$
\end{tabular}

$\mathrm{RR}=$ relative risk; $\mathrm{CI}=$ confidence interval; $\mathrm{MA}=$ Mexican American; NHW = non-Hispanic white; NIHSS = National Institutes of Health Stroke Scale. 
diabetes and coronary artery disease and indicates that, in both ethnic groups, secondary stroke prevention is critical to improving survival after ischemic stroke.

Interestingly, in the current model predicting allcause mortality, MAs had better survival than NHWs after ischemic stroke adjusted for confounders and independent predictors of mortality, despite the increased risk for recurrent stroke in MAs and similar impact of recurrent stroke on mortality in the two ethnic groups. The combination of the current findings and our previous finding of decreased mortality after ischemic stroke in $\mathrm{MAs}^{17}$ suggests that there are unidentified factors that contribute to better survival in the MA population. Perhaps differential social support and/or genetic factors within ethnic groups may relate to this ethnic association with mortality.

Limitations of this work warrant discussion. Survival estimates from the stratified analysis were somewhat imprecise due to the small sample size within strata, but they are nonetheless helpful in trying to hypothesize about the nature of the relation between ethnicity and recurrent stroke. The association between ethnicity and recurrence may have been the result of residual confounding or factors unaccounted for in the multivariable model. For example, we did not adjust for stroke subtype. Ischemic stroke subtype has been shown to influence risk for stroke recurrence in some studies, ${ }^{4,8,26,27}$ but not others. ${ }^{6,28-30}$ We have previously demonstrated that stroke subtype does not differ by ethnicity in this population, suggesting this factor does not confound the observed association between ethnicity and stroke recurrence. ${ }^{31}$ Furthermore, in a subset of the data for this study for which ischemic stroke subtype was available $(\mathrm{n}=250)$, subtype was not related to recurrent stroke $(p=0.3238)$, again suggesting it is an unlikely confounder. Use of or compliance with secondary stroke prevention medications may influence risk for recurrent stroke. We have demonstrated previously that among ischemic stroke cases in this population, there were no ethnic differences in the proportion that report taking medications for stroke risk factors or other secondary stroke prevention medications. ${ }^{14}$ Nonetheless, it is unclear whether differences may exist in control of traditional vascular risk factors, such as diabetes and hypertension, in MAs and NHWs. Differences in physician treatment, patient compliance, or differential drug effectiveness by ethnicity could also translate into increased stroke recurrence for MAs. In our multivariable models, we adjusted for potential confounders but did not explore possible interactions in these variables due to the limited number of outcome events. It would also be of interest to understand how these factors may interact to influence recurrence, mortality, or both. It is possible that some recurrent strokes may have been missed. In this analysis, recurrent strokes were identified among subsequent hospital admissions, emergency department visits, or both. It is possible that an individual may have experienced a new stroke during his or her initial hospitalization that was not captured, although this is unlikely. In addition, if individuals sought medical care outside of the study county for a recurrent stroke, the event may have been missed; however, the study community is stable with minimal efflux. ${ }^{18}$ In our analysis of a convenience sample of deaths among ischemic stroke cases, we found the frequency of recurrent strokes missed by our surveillance methods to be small at 2 to $3 \%$ with no difference by ethnicity. Therefore, our estimates of risk for recurrence may be underestimated, but the finding of an ethnic difference in risk for recurrence was likely not affected by ascertainment bias. In this analysis, recurrent strokes were the only vascular events recorded. It would also be of interest to investigate the rate of other vascular events after stroke, and this should be considered in future studies. Caution should be taken when generalizing these estimates to other communities, which may differ by ethnic composition or sociodemographic structure.

In summary, MAs had higher stroke recurrence risk compared with NHWs; however, recurrent stroke influenced all-cause mortality to a similar extent across ethnic groups. These findings add to the literature regarding the burden of stroke in MAs, the fastest growing segment of the US population. Efforts to reduce stroke recurrence in MAs are needed.

This study was funded by the NIH (National Institute on Neurological Disorders and Stroke, RO1 NS38916, L.B.M.).

\section{References}

1. American Heart Association. Heart disease and stroke statistics-2005 update. Dallas, TX: American Heart Association, 2004.

2. Sacco RL, Foulkes MA, Mohr JP, et al. Determinants of early recurrence of cerebral infarction. The Stroke Data Bank. Stroke 1989;20:983-989.

3. Sacco RL, Shi T, Zamanillo MC, Kargman DE. Predictors of mortality and recurrence after hospitalized cerebral infarction in an urban community: the Northern Manhattan Stroke Study. Neurology 1994;44:626-634.

4. Modrego PJ, Mainar R, Turull L. Recurrence and survival after first-ever stroke in the area of Bajo Aragon, Spain. A prospective cohort study. J Neurol Sci 2004;224:49-55.

5. Petty GW, Brown RD Jr, Whisnant JP, et al. Survival and recurrence after first cerebral infarction: a population-based study in Rochester, Minnesota, 1975 through 1989. Neurology 1998; 50:208-216.

6. Burn J, Dennis M, Bamford J, et al. Long-term risk of recurrent stroke after a first-ever stroke. The Oxfordshire Community Stroke Project. Stroke 1994;25:333-337.

7. Ryglewicz D, Baranska-Gieruszczak M, Czlonkowska A, et al. Stroke recurrence among 30 days survivors of ischemic stroke in a prospective community-based study. Neurol Res 1997;19: 377-379. 
8. Yokota C, Minematsu K, Hasegawa Y, Yamaguchi T. Longterm prognosis, by stroke subtypes, after a first-ever stroke: a hospital-based study over a 20-year period. Cerebrovasc Dis 2004;18:111-116.

9. Appelros P, Nydevik I, Viitanen M. Poor outcome after firstever stroke: predictors for death, dependency, and recurrent stroke within the first year. Stroke 2003;34:122-126.

10. Moroney JT, Bagiella E, Paik MC, et al. Risk factors for early recurrence after ischemic stroke: the role of stroke syndrome and subtype. Stroke 1998;29:2118-2124.

11. Jorgensen HS, Nakayama H, Reith J, et al. Stroke recurrence: predictors, severity, and prognosis. The Copenhagen Stroke Study. Neurology 1997;48:891-895.

12. Hankey GJ, Jamrozik K, Broadhurst RJ, et al. Long-term disability after first-ever stroke and related prognostic factors in the Perth Community Stroke Study, 1989-1990. Stroke 2002;33: $1034-1040$.

13. Sacco RL, Boden-Albala B, Gan R, et al. Stroke incidence among white, black, and Hispanic residents of an urban community: the Northern Manhattan Stroke Study. Am J Epidemiol 1998;147:259-268.

14. Morgenstern LB, Smith MA, Lisabeth LD, et al. Excess stroke in Mexican Americans compared with non-Hispanic Whites: the Brain Attack Surveillance in Corpus Christi Project. Am J Epidemiol 2004; 160:376-383.

15. Sheinart KF, Tuhrim S, Horowitz DR, et al. Stroke recurrence is more frequent in Blacks and Hispanics. Neuroepidemiology 1998;17:188-198.

16. Smith MA, Risser JM, Lisabeth LD, et al. Access to care, acculturation, and risk factors for stroke in Mexican Americans: the Brain Attack Surveillance in Corpus Christi (BASIC) project. Stroke 2003;34:2671-2675.

17. Lisabeth LD, Risser JM, Brown DL, et al. Stroke burden in Mexican Americans: the impact of mortality following stroke. Ann Epidemiol 2006;16:33-40.

18. Piriyawat P, Smajsova M, Smith MA, et al. Comparison of active and passive surveillance for cerebrovascular disease: the Brain Attack Surveillance in Corpus Christi (BASIC) project. Am J Epidemiol 2002;156:1062-1069.

19. Gillum RF, Fortmann SP, Prineas RJ, Kottke TE. International diagnostic criteria for acute myocardial infarction and acute stroke. Am Heart J 1984;108:150-158.
20. Centers for Disease Control and Prevention/Center for Health Statistics. Instructions for classifying underlying cause-of-death, ICD-10, 2005. Available at: http://www.cdc.gov/nchs/about/ major/dvs/im.htm. Accessed Jun 27, 2005.

21. Asplund K, Tuomilehto J, Stegmayr B, et al. Diagnostic criteria and quality control of the registration of stroke events in the MONICA project. Acta Med Scand Suppl 1988;728:26-39.

22. Smith MA, Risser JM, Moye LA, et al. Designing multi-ethnic stroke studies: the Brain Attack Surveillance in Corpus Christi (BASIC) project. Ethn Dis 2004;14:520-526.

23. Williams LS, Yilmaz EY, Lopez-Yunez AM. Retrospective assessment of initial stroke severity with the NIH Stroke Scale. Stroke 2000;31:858-862.

24. Lauria G, Gentile M, Fassetta G, et al. Incidence and prognosis of stroke in the Belluno province, Italy. First-year results of a community-based study. Stroke 1995;26:1787-1793.

25. Lee AH, Somerford PJ, Yau KK. Risk factors for ischaemic stroke recurrence after hospitalisation. Med J Aust 2004;181: $244-246$.

26. Hata J, Tanizaki Y, Kiyohara Y, et al. Ten year recurrence after first ever stroke in a Japanese community: the Hisayama study. J Neurol Neurosurg Psychiatry 2005;76:368-372.

27. Lovett JK, Coull AJ, Rothwell PM. Early risk of recurrence by subtype of ischemic stroke in population-based incidence studies. Neurology 2004;62:569-573.

28. Kolominsky-Rabas PL, Weber M, Gefeller O, et al. Epidemiology of ischemic stroke subtypes according to TOAST criteria: incidence, recurrence, and long-term survival in ischemic stroke subtypes: a population-based study. Stroke 2001;32:2735-2740.

29. Hillen T, Coshall C, Tilling K, et al. Cause of stroke recurrence is multifactorial: patterns, risk factors, and outcomes of stroke recurrence in the South London Stroke Register. Stroke 2003; 34:1457-1463.

30. Murat Sumer M, Erturk O. Ischemic stroke subtypes: risk factors, functional outcome and recurrence. Neurol Sci 2002;22: 449-454.

31. Uchino K, Risser JM, Smith MA, et al. Ischemic stroke subtypes among Mexican Americans and non-Hispanic whites: the BASIC Project. Neurology 2004;63:574-576. 